Article

\title{
Genetic Analyses Confirm SNPs in HSPA8 and ERBB2 are Associated with Milk Protein Concentration in Chinese Holstein Cattle
}

\author{
Cong $\mathrm{Li}^{1,+}{ }^{+}$, Miao Wang ${ }^{1,+}$, Wentao Cai $\left.{ }^{2}{ }^{(}\right)$, Shuli Liu ${ }^{2}$, Chenghao Zhou ${ }^{2}$, Hongwei Yin ${ }^{2}$, \\ Dongxiao Sun ${ }^{2}$ and Shengli Zhang ${ }^{2, *}$ \\ 1 Shaanxi Key Laboratory of Molecular Biology for Agriculture, College of Animal Science and Technology, \\ Northwest A\&F University, Yangling, Shaanxi 712100, China; congl@nwafu.edu.cn (C.L.); \\ wangmiao9254@gmail.com (M.W.) \\ 2 Department of Animal Genetics and Breeding, College of Animal Science and Technology, Key Laboratory \\ of Animal Genetics and Breeding of Ministry of Agriculture, National Engineering Laboratory for Animal \\ Breeding, China Agricultural University, Beijing 100193, China; wtaocai@163.com (W.C.); \\ shuliliu1991@cau.edu.cn (S.L.); reddsbhj3@163.com (C.Z.); yinhongwei@cau.edu.cn (H.Y.); \\ sundx@cau.edu.cn (D.S.) \\ * Correspondence: zhangslcau@cau.edu.cn; Tel.: +86-13901194331; Fax: +86-01062733697 \\ + These authors contributed equally to this work.
}

Received: 28 November 2018; Accepted: 28 January 2019; Published: 30 January 2019

check for updates

\begin{abstract}
Heat shock $70 \mathrm{kDa}$ protein 8 (HSPA8) and erb-b2 receptor tyrosine kinase 2 (ERBB2) were the promising candidates for milk protein concentration in dairy cattle revealed through previous RNA sequencing (RNA-Seq) study. The objective of this post-RNA-Seq study was to confirm genetic effects of $H S P A 8$ and ERBB2 on milk protein concentration in a large Chinese Holstein population and to evaluate the genetic effects of both genes on other milk production traits. There were 2 single-nucleotide polymorphisms (SNPs) identified for HSPA8 and 11 SNPs for ERBB2 by sequencing 17 unrelated Chinese Holstein sires. The SNP-rs136632043 in HSPA8 had significant associations with all five milk production traits $(p=0.0086$ to $p<0.0001)$, whereas SNP-rs132976221 was remarkably associated with three yield traits $(p<0.0001)$. Nine (ss1996900615, rs109017161, rs109122971, ss1996900614, rs110133654, rs109941438, rs110552983, rs133031530, and rs109763505) of 11 SNPs in ERBB2 were significantly associated with milk protein percentage ( $p=0.0177$ to $p<0.0001$ ). A $12 \mathrm{~Kb}$ haplotype block was formed in $E R B B 2$ and haplotype associations revealed similar effects on milk protein traits. Our findings confirmed the significant genetic effects of HSPA8 and ERBB2 on milk protein concentration and other milk production traits and SNP phenotypic variances above $1 \%$ may serve as genetic markers in dairy cattle breeding programs.
\end{abstract}

Keywords: candidate genes; milk protein traits; association analyses; haplotypes; dairy cows

\section{Introduction}

Molecular selective breeding strategies have been widely applied in animal breeding to improve the important economic traits of livestock. Identification of key genes or causal variations for economic traits is prerequisite to perform molecular selective breeding strategies [1,2]. Milk protein concentration is an important index to evaluate the nutritional value in cow's milk. However, limited key genes or variations for milk protein concentration were found in dairy cattle [3-9]. Our initial RNA sequencing (RNA-Seq) study revealed that heat shock $70 \mathrm{kDa}$ protein 8 (HSPA8) and erb-b2 receptor tyrosine kinase 2 (ERBB2) were candidate genes affecting milk protein traits in dairy cows [10]. It was observed that HSPA8 $\left(\log _{2}\right.$ fold change $=-1.13, q$-value $\left.=2.91 \times 10^{-2}\right)$ and ERBB2 $\left(\log _{2}\right.$ fold change $=1.00$, 
$q$-value $=5.25 \times 10^{-3}$ ) were significant differentially expressed in bovine mammary tissues of cows in high and low milk protein percentage comparisons [10].

HSPA 8 is located in BTA15 with a total length of $4426 \mathrm{bp}$, containing 9 exons and 8 introns, and encoding 650 amino acids. HSPA8 is an important gene in the MAPK pathway [11], which has a positive effect on protein synthesis by increasing the stability of mRNA through phosphorylation of the AU-rich element-binding protein [12]. HSPA8 is highly expressed in the lactating mammary gland $[10,13]$, which has a role in regulating protein folding and processing in the endoplasmic reticulum [14], likely in support of active milk protein synthesis [13]. ERBB2 encodes a receptor of tyrosine kinases [15] and is located in BTA19 with a total length of 24,682 bp, containing 27 exons and 26 introns, and encoding 1255 amino acids. ERBB2 could activate PI3K signaling pathway by directly binding of PI3K regulatory subunit $\mathrm{p} 85$ to phosphorylated tyrosine residues, which is known to regulate milk protein synthesis [16]. Most importantly, HSPA8 is known to be downstream of ERBB2 in human $[17,18]$, indicating the probably regulatory effect of ERBB2 to HSPA 8 and working together in specific biological processes.

Genetic analyses between selected genes and bovine milk production traits can provide valuable molecular information for the genetic improvement program for milk quality of dairy cattle. Therefore, the aims of this study were to identify genetic polymorphisms in HSPA8 and ERBB2, to explore the linkages among single-nucleotide polymorphisms (SNPs), and to conduct the genetic effects analyses in a large Chinese Holstein population.

\section{Materials and Methods}

\subsection{Animal Population and Phenotypic Data}

A total of 1027 Chinese Holstein cows from 17 sire families were used to construct the study population. Family size ranges from 25 to 187 daughters with an average of 60 daughters per sire (Table S1). Cows were selected from 17 dairy farms in the Beijing Sanyuan Lvhe Dairy Farm Center, where regular and standard performance testing (Dairy Herd Improvement, DHI) have been implemented since 1999. Five milk production traits ( $305 \mathrm{~d}$ milk yield, $305 \mathrm{~d}$ protein yield, $305 \mathrm{~d}$ fat yield, average $305 \mathrm{~d}$ protein percentage, and average $305 \mathrm{~d}$ fat percentage) were collected from individual animal via the complete DHI data and were used for the subsequent analyses.

\subsection{Genomic DNA Extraction}

Blood samples were collected from 1027 Chinese Holstein cows via coccygeal vein and stored at $-20^{\circ} \mathrm{C}$. The tubule frozen semen samples of 17 sires were collected from Beijing Bull Station. Genomic DNA was extracted from blood samples with a TIANamp Blood DNA kit (TIANGEN Biotech, Beijing, China) and from the semen samples using a standard phenol-chloroform procedure [19]. The quantity and quality of isolated DNA were confirmed before further analysis.

\subsection{SNP Identification and Genotyping}

A DNA pool was constructed from aforementioned 17 Holstein bulls ( $50 \mathrm{ng} / \mathrm{uL}$ of each individual) to identify potential SNPs that were involved in HSPA8 and ERBB2 genes. A total of 13 and 26 pairs of primers (Table S2) were designed to amplify all exons and their partial flanking intronic sequences based on the reference sequences of the bovine HSPA8 (NCBI Reference Sequence: AC_000172.1) and ERBB2 (NCBI Reference Sequence: AC_000176.1) referring to Bos_taurus_UMD_3.1 assembly using Primer3 web Program v.0.4.0 (http:/ / primer3.ut.ee), respectively.

The polymerase chain reaction (PCR) was performed to amplify the pooled DNA from 17 sires with a final reaction volume of $25 \mu \mathrm{L}$, comprising of $50 \mathrm{ng}$ genomic DNA, $0.5 \mu \mathrm{L}$ of each primer, $2.5 \mu \mathrm{L}$ $10 \times$ PCR buffer, $2.5 \mathrm{mM}$ each of dNTP, and $1 \mathrm{U}$ of Taq DNA polymerase (Takara Biotechnology Co., Ltd., Dalian, China). The PCR protocol was $5 \mathrm{~min}$ at $94{ }^{\circ} \mathrm{C}$ for initial denaturing, followed by 34 cycles at $94{ }^{\circ} \mathrm{C}$ for $30 \mathrm{~s}, 56{ }^{\circ} \mathrm{C}$ for $30 \mathrm{~s}, 72{ }^{\circ} \mathrm{C}$ for $30 \mathrm{~s}$, and a final extension at $72{ }^{\circ} \mathrm{C}$ for $7 \mathrm{~min}$. The amplification 
products were visualized by gel electrophoresis on $2 \%$ agarose gels, followed by photography under UV light. After that, $40 \mu \mathrm{L}$ of each PCR product from the pooled DNA was bi-directionally sequenced using the ABI3730XL (Applied Biosystems, Foster City, CA, USA), and the sequences were aligned to the bovine reference sequences (UMD3.1) using BLAST (http:/ /blast.ncbi.nlm.nih.gov/Blast.cgi) to identify potential SNPs. The subsequent genotyping analysis of the 1027 Chinese Holstein cows were performed with matrix-assisted laser desorption/ionization time of flight mass spectrometry (MALDI-TOF MS, Squenom MassARRAY, Bioyong Technologies Inc. HK) assay.

\subsection{Linkage Disequilibrium Analysis}

Pair-wise linkage disequilibrium (LD) was measured for each pair of SNPs genotyped within the HSPA8 and ERBB2 genes based on the criterion of $\mathrm{D}$ prime $\left(\mathrm{D}^{\prime}\right)$ using Haploview [20]. Genotypes were firstly imputed for each individual using the Beagle3.2 software program [21]. Briefly, an iterative algorithm was applied for fitting a haplotype Hidden Markov Model (HMM) to genotype data that alternated between model building and sampling. In the model-building step, current estimates of phased haplotypes are used for building a new haplotype HMM, in the sampling step, new haplotypes are sampled for each individual conditional upon the genotype data and current haplotype HMM. Estimated phased haplotypes for the initial iteration are obtained by imputing missing genotypes at random according to allele frequencies and randomly phasing heterozygous genotypes. Accordingly, haplotype blocks where SNPs are in high LD $\left(\mathrm{D}^{\prime}>0.90\right)$ were determined based on confidence intervals methods [22]. A haplotype with a frequency $>5 \%$ was considered as a distinguishable haplotype, while the haplotypes with relative frequency $<5 \%$ were pooled into a single group. Haplotype blocks within relative SNPs were applied to subsequent analyses to detect their associations with phenotypes.

\subsection{Association and Haplotype Analyses}

Hardy-Weinberg equilibrium test was conducted on each identified SNP. Chi-square was used to compare the number of expected and observed genotypes with a significance level of 0.05 . The genetic effects of each candidate SNP or haplotype on five milk production traits were analyzed with the mixed procedure of SAS (SAS Institute Inc., Cary, NC, USA) with the following statistical model:

$$
\mathrm{y}_{\mathrm{ijklmn}}=\mu+F_{i}+Y S_{j}+P_{k}+\mathrm{b} \times \mathrm{M}+G_{l}+\alpha_{\mathrm{m}}+\mathrm{e}_{\mathrm{ijklmn}}
$$

where, $\mathrm{y}_{\mathrm{ijk} k \mathrm{mn}}$ was the phenotypic value of each trait of cows $(n=1027$ for each trait); $\mu$ was the overall mean; $F_{i}$ was the fixed effect of farm; $Y S_{j}$ was the fixed effect of year-season; $P_{k}$ was the fixed effect of parity; $M$ was the covariate effect of calving month; $b$ was the regression coefficient of $M$; $G_{l}$ was the fixed effect corresponding to the genotype of polymorphisms or haplotype; $\alpha_{\mathrm{m}}$ was the random polygenic effect, distributed as $\mathrm{N}\left(0, \mathrm{A \sigma}_{\mathrm{a}}{ }^{2}\right)$, with the additive genetic relationship matrix $\mathrm{A}$ and the additive genetic variance $\sigma_{\mathrm{a}}{ }^{2}$; and $\mathrm{e}_{\mathrm{ijk} k \mathrm{mn}}$ was the random residual, distributed as $\mathrm{N}\left(0, \mathrm{I}_{\mathrm{e}}{ }^{2}\right)$, with identity matrix I and residual error variance $\sigma_{\mathrm{e}}{ }^{2}$. The overall reliability of the whole model was symbolized as 'Goodness of Fit', and was calculated by $R^{2}$ as following formula,

$$
R^{2}=S S_{R} / S S_{T}=1-S S_{E} / S S_{T}=1-\sum\left(\text { Residual }^{2}\right) / \sum(Y-\bar{Y})^{2}
$$

where, $R^{2}$ was the overall reliability; $S S_{R}$ was sum of squares of variables; $S S_{T}$ was total sum of squares; $S S_{E}$ was residual sum of squares; Residual was the residuals; $Y$ was phenotypes of traits; $\bar{Y}$ was mean values of phenotypes of traits. The results showed that the $R^{2}$ of milk yield, milk protein percentage, milk fat percentage, milk protein yield and milk fat yield are $0.55,0.50,0.66,0.73,0.76$, respectively, suggests that the model provide a good fit.

The differences among the effects of single SNPs or haplotypes on each trait were compared with Bonferroni correction. The significant level of the multiple tests was equal to the raw $P$ value divided by number of tests. The additive (a), dominance (d) and allele substitution ( $\alpha$ ) effects were 
estimated according to the equation proposed by Falconer \& Mackay [23], i.e., a $=(\mathrm{AA}-\mathrm{BB}) / 2$, $\mathrm{d}=\mathrm{AB}-(\mathrm{AA}+\mathrm{BB}) / 2$ and $\alpha=\mathrm{a}+\mathrm{d}(\mathrm{q}-\mathrm{p})$, where $\mathrm{AA}$ and $\mathrm{BB}$ represent the two homozygous genotypes, $A B$ is heterozygous genotype, and $p$ and $q$ are the allele frequencies of corresponding loci.

\subsection{Phenotypic Variance}

The proportion of phenotypic variance of the trait explained by a SNP was symbolized to show the effect of a SNP on a specific trait. The calculation formula is:

$$
\text { Phenotypic variance ratio }=2 p(1-p) \alpha^{2} / \sigma_{p}^{2}
$$

where $p$ is the allele frequency of SNP, $\alpha$ is the average effect of gene substitution calculated by the linear mixed model, and $\sigma_{p}^{2}$ is the estimate of the phenotypic variance using the complete DHI data of Chinese dairy cattle population.

\subsection{Ethics Approval and Consent to Participate}

All protocols for collection of the blood and frozen semen samples of experimental individuals were approved by the Institutional Animal Care and Use Committee (IACUC) at China Agricultural University (Permit Number: DK996). We obtained written agreements from the cattle owners to use the samples and data.

\section{Results}

\subsection{SNPs Identification}

Two SNPs of rs136632043 and rs132976221 were identified for HSPA8 gene, with one located in the $3^{\prime}$ regulatory region $\left(3^{\prime}\right.$-UTR) and the other located in the intron (Table 1). A total of 11 SNPs was discovered for ERBB2 gene. Among these identified SNPs, eight SNPs (ss1996900615, rs109122971, ss1996900614, rs110133654, rs109941438, rs110552983, rs133031530 and rs109763505) were found within introns, one (rs133724008) was in the $5^{\prime}$ regulatory region ( $5^{\prime}$-UTR), and two SNPs (rs110735562 and rs109017161) were synonymous substitutions located in exons. All 13 identified SNPs of HSPA8 and ERBB2 genes were in Hardy-Weinberg equilibrium $(p>0.05$, Table 2).

Table 1. Information for the identified single-nucleotide polymorphisms (SNPs) in HSPA8 and ERBB2 genes.

\begin{tabular}{|c|c|c|c|c|c|c|}
\hline CHR & RefSNP & SNP Locus & Alleles & Location & Position & Gene \\
\hline 15 & rs132976221 & g. $1585 A>C$ & $\mathrm{~A} / \mathrm{C}$ & Intron-3 & 34219120 & HSPA8 \\
\hline 15 & rs136632043 & g.4218T $>\mathrm{G}$ & $\mathrm{T} / \mathrm{G}$ & 3'-UTR & 34216487 & HSPA8 \\
\hline 19 & rs133724008 & g. $873 \mathrm{~T}>\mathrm{C}$ & $\mathrm{T} / \mathrm{C}$ & 5'-UTR & 40720963 & $E R B B 2$ \\
\hline 19 & ss1996900615 & g.20982del & $\mathrm{TC} /$. & Intron-19 & 40742818 & $E R B B 2$ \\
\hline 19 & rs110735562 & g. $21561 \mathrm{~A}>\mathrm{G}$ & $\mathrm{A} / \mathrm{G}$ & Exon-21 & 40743397 & $E R B B 2$ \\
\hline 19 & rs109017161 & g.22268T >C & $\mathrm{T} / \mathrm{C}$ & Exon-23 & 40744104 & $E R B B 2$ \\
\hline 19 & rs109122971 & g.23650T $>C$ & $\mathrm{~T} / \mathrm{C}$ & Intron-26 & 40745486 & $E R B B 2$ \\
\hline 19 & ss1996900614 & g.19414A > G & $\mathrm{A} / \mathrm{G}$ & Intron-14 & 40741250 & $E R B B 2$ \\
\hline 19 & rs110133654 & g.10727A $>G$ & $\mathrm{~A} / \mathrm{G}$ & Intron-7 & 40732563 & $E R B B 2$ \\
\hline 19 & rs109941438 & g.11680C $>$ T & $\mathrm{C} / \mathrm{T}$ & Intron-8 & 40733516 & $E R B B 2$ \\
\hline 19 & rs110552983 & g. $16431 C>G$ & $\mathrm{C} / \mathrm{G}$ & Intron-14 & 40738267 & $E R B B 2$ \\
\hline 19 & rs133031530 & g. $22346 \mathrm{~A}>\mathrm{T}$ & $\mathrm{A} / \mathrm{T}$ & Intron-23 & 40744182 & $E R B B 2$ \\
\hline 19 & rs109763505 & g. $22400 A>G$ & $\mathrm{~A} / \mathrm{G}$ & Intron-23 & 40744236 & $E R B B 2$ \\
\hline
\end{tabular}


Table 2. Genotypic and allelic frequencies and Hardy-Weinberg equilibrium test of SNPs of HSPA8 and ERBB2 genes in Chinese Holstein cattle.

\begin{tabular}{|c|c|c|c|c|c|c|c|c|}
\hline Gene & Position & Locus & Genotypes & $\mathbf{N}$ & Frequency & Allele & Frequency & $\begin{array}{l}\text { Hardy-Weinberg } \\
\text { Equilibrium } \chi^{2} \text { Test }\end{array}$ \\
\hline \multirow{3}{*}{ HSPA8 } & \multirow{3}{*}{ Intron-3 } & \multirow{3}{*}{$\begin{array}{l}\text { rs } 132976221 \\
\text { g.1585A }>C\end{array}$} & CA & 432 & 0.428 & A & 0.702 & \multirow{3}{*}{$p>0.05$} \\
\hline & & & AA & 493 & 0.488 & $\mathrm{C}$ & 0.298 & \\
\hline & & & $\mathrm{CC}$ & 85 & 0.084 & & & \\
\hline \multirow{3}{*}{ HSPA8 } & \multirow{3}{*}{$\begin{array}{l}3^{\prime} \text { prime } \\
\text { UTR (exon9) }\end{array}$} & \multirow{3}{*}{$\begin{array}{c}\text { rs136632043 } \\
\text { g.4218T }>G\end{array}$} & GT & 277 & 0.274 & G & 0.175 & \multirow{3}{*}{$p>0.05$} \\
\hline & & & GG & 38 & 0.038 & $\mathrm{~T}$ & 0.825 & \\
\hline & & & TT & 696 & 0.688 & & & \\
\hline \multirow{3}{*}{ ERBB2 } & \multirow{3}{*}{$\begin{array}{l}5^{\prime} \text { flanking } \\
\text { region }\end{array}$} & \multirow{3}{*}{$\begin{array}{c}\text { rs133724008 } \\
\text { g.873T >C }\end{array}$} & $\mathrm{CT}$ & 412 & 0.411 & $\mathrm{C}$ & 0.324 & \multirow{3}{*}{$p>0.05$} \\
\hline & & & $\mathrm{CC}$ & 119 & 0.119 & $\mathrm{~T}$ & 0.676 & \\
\hline & & & TT & 471 & 0.470 & & & \\
\hline \multirow{3}{*}{ ERBB2 } & \multirow{3}{*}{ Intron-19 } & \multirow{3}{*}{$\begin{array}{l}\text { ss1996900615 } \\
\text { g.20982del }\end{array}$} & DEL.TC & 471 & 0.469 & DEL & 0.604 & \multirow{3}{*}{$p>0.05$} \\
\hline & & & DEL & 372 & 0.370 & $\mathrm{TC}$ & 0.396 & \\
\hline & & & TC & 162 & 0.161 & & & \\
\hline \multirow{3}{*}{ ERBB2 } & \multirow{3}{*}{ Exon-21 } & \multirow{3}{*}{$\begin{array}{l}\text { rs } 110735562 \\
\text { g.21561A }>\mathrm{G}\end{array}$} & AG & 362 & 0.358 & A & 0.243 & \multirow{3}{*}{$p>0.05$} \\
\hline & & & AA & 65 & 0.064 & G & 0.757 & \\
\hline & & & GG & 585 & 0.578 & & & \\
\hline \multirow{3}{*}{ ERBB2 } & \multirow{3}{*}{ Exon-23 } & \multirow{3}{*}{$\begin{array}{l}\text { rs109017161 } \\
\text { g.22268T>C }\end{array}$} & $\mathrm{CT}$ & 458 & 0.458 & $\mathrm{C}$ & 0.607 & \multirow{3}{*}{$p>0.05$} \\
\hline & & & $\mathrm{CC}$ & 378 & 0.378 & $\mathrm{~T}$ & 0.393 & \\
\hline & & & $\mathrm{TT}$ & 164 & 0.164 & & & \\
\hline \multirow{3}{*}{ ERBB2 } & & & $\mathrm{CT}$ & 473 & 0.473 & $\mathrm{C}$ & 0.568 & \\
\hline & Intron-26 & $\begin{array}{l}\operatorname{rs} 109122971 \\
\text { g.23650T }>C\end{array}$ & $\mathrm{CC}$ & 332 & 0.332 & $\mathrm{~T}$ & 0.432 & $p>0.05$ \\
\hline & & & $\mathrm{TT}$ & 196 & 0.196 & & & \\
\hline & & ss1996900614 & AG & 470 & 0.463 & A & 0.394 & \\
\hline$E R B B 2$ & Intron-14 & $\begin{array}{c}\text { SS1996900b14 } \\
\text { g.19414A>CG }\end{array}$ & AA & 165 & 0.162 & G & 0.606 & $p>0.05$ \\
\hline & & & GG & 381 & 0.375 & & & \\
\hline & & rs110133654 & AG & 470 & 0.468 & A & 0.604 & \\
\hline ERBB2 & Intron-7 & g.10727A $>G$ & AA & 372 & 0.370 & G & 0.396 & $p>0.05$ \\
\hline & & & GG & 163 & 0.162 & & & \\
\hline & & & $\mathrm{CT}$ & 452 & 0.455 & $\mathrm{C}$ & 0.605 & \\
\hline ERBB2 & Intron-8 & $\begin{array}{l}\text { rs109941438 } \\
\text { g.11680C }>\mathrm{T}\end{array}$ & $\mathrm{CC}$ & 375 & 0.378 & $\mathrm{~T}$ & 0.395 & $p>0.05$ \\
\hline & & & $\mathrm{TT}$ & 166 & 0.167 & & & \\
\hline & & & CG & 472 & 0.464 & $\mathrm{C}$ & 0.393 & \\
\hline$E R B B 2$ & Intron-14 & g. $16431 C>G$ & $\mathrm{CC}$ & 164 & 0.161 & G & 0.607 & $p>0.05$ \\
\hline & & & GG & 381 & 0.375 & & & \\
\hline & & & AT & 445 & 0.451 & A & 0.390 & \\
\hline$E R B B 2$ & Intron-23 & g. $22346 \mathrm{~A}>\mathrm{T}$ & AA & 162 & 0.164 & $\mathrm{~T}$ & 0.610 & $p>0.05$ \\
\hline & & & $\mathrm{TT}$ & 379 & 0.384 & & & \\
\hline & & & AG & 483 & 0.478 & A & 0.432 & \\
\hline$E R B B 2$ & Intron-23 & g. $22400 A>G$ & AA & 195 & 0.193 & G & 0.568 & $p>0.05$ \\
\hline & & & GG & 332 & 0.329 & & & \\
\hline
\end{tabular}

\subsection{Single Locus-Based Association Analyses}

SNP-rs136632043 was highly associated with all five milk production traits $(p=0.0086$ to $p<0.0001$; Table 3). SNP-rs132976221 also showed strong associations with three yield traits (milk yield, fat yield, and protein yield, $p<0.0001$ ). There were six significant pairs of SNP-trait explaining phenotypic variations with greater than $1 \%$, a range from 1.70 to $5.13 \%$ (Table 3). In addition, SNPs in HSPA8 also showed the corresponding significant additive, substitution, or dominant effects on target traits (Table S3).

Nine SNPs (ss1996900615, rs109017161, rs109122971, ss1996900614, rs110133654, rs109941438, rs110552983, rs133031530, and rs109763505) in ERBB2 were significantly associated with milk protein percentage ( $p=0.0177$ to $p<0.0001$; Table 3). SNPs of rs110735562 and rs133724008 had significant associations with three yield traits (milk yield, fat yield, and protein yield, $p=0.0290$ to $p<0.0001$ ), whereas SNP of rs133724008 was also significantly associated with milk protein percentage $(p<0.0001$; Table 3). Phenotypic variations explained by the 11 SNPs in ERBB2 with greater than $1 \%$ were existed in four significant pairs of SNP-trait, ranging from 1.49 to 2.05\% (Table 3). Nine SNPs (ss1996900615, rs109017161, rs109122971, ss1996900614, rs110133654, rs109941438, rs110552983, rs133031530, and rs109763505) had significant dominant effects on milk protein percentages (Table S3). SNP-rs110735562 had significant dominant effects on three yield traits. SNP-rs133724008 showed significant additive 
and substitution effects on three milk yield traits and significant dominant effects on milk protein percentage (Table S3).

Table 3. Associations of identified SNPs of HSPA 8 and ERBB2 genes with milk production traits in Chinese Holstein cattle (Least square mean \pm Standard error, $\mathrm{LSM} \pm \mathrm{SE}$ ).

\begin{tabular}{|c|c|c|c|c|c|c|}
\hline Locus & Genotypes & Milk Yield & Fat Yield & Fat Percentage & Protein Yield & Protein Percentage \\
\hline \multirow{5}{*}{$\begin{array}{c}\text { HSPA8 } \\
\text { rs132976221 } \\
\text { g.1585A }>C\end{array}$} & AA(493) & $10307 \pm 61.79 \mathrm{~A}$ & $361.58 \pm 2.58^{\mathrm{A}}$ & $3.572 \pm 0.025$ & $323.23 \pm 1.88^{\mathrm{A}}$ & $3.169 \pm 0.009$ \\
\hline & $\mathrm{AC}(432)$ & $10639 \pm 62.63^{\text {B }}$ & $373.69 \pm 2.62^{B}$ & $3.580 \pm 0.025$ & $333.02 \pm 1.91^{\text {B }}$ & $3.164 \pm 0.009$ \\
\hline & $\mathrm{CC}(85)$ & $10465 \pm 100.55^{\mathrm{AB}}$ & $367.58 \pm 4.24 \mathrm{AB}$ & $3.576 \pm 0.041$ & $330.38 \pm 3.09^{\text {B }}$ & $3.180 \pm 0.014$ \\
\hline & $p$-value & $<0.0001$ & $<0.0001$ & 0.9436 & $<0.0001$ & 0.4773 \\
\hline & Variance & $4.76 \times 10^{-2}$ & $3.74 \times 10^{-2}$ & $1.48 \times 10^{-4}$ & $5.13 \times 10^{-2}$ & $8.83 \times 10^{-5}$ \\
\hline \multirow{5}{*}{$\begin{array}{c}\text { HSPA8 } \\
\text { rs } 136632043 \\
\text { g.4218T>G }\end{array}$} & GG(38) & $10632 \pm 140.30^{\mathrm{A}}$ & $403.60 \pm 5.93^{\mathrm{A}}$ & $3.797 \pm 0.057^{\mathrm{A}}$ & $338.32 \pm 4.32 \mathrm{~A}$ & $3.192 \pm 0.020^{\mathrm{A}}$ \\
\hline & GT(277) & $10339 \pm 67.43^{\mathrm{B}}$ & $363.93 \pm 2.82^{B}$ & $3.586 \pm 0.027^{\mathrm{B}}$ & $325.44 \pm 2.06^{B}$ & $3.188 \pm 0.010^{\mathrm{A}}$ \\
\hline & ТT(696) & $10532 \pm 59.56^{\mathrm{A}}$ & $364.61 \pm 2.48^{B}$ & $3.536 \pm 0.024^{\mathrm{B}}$ & $328.70 \pm 1.81^{\mathrm{B}}$ & $3.154 \pm 0.008^{B}$ \\
\hline & $p$-value & 0.0022 & $<0.0001$ & $<0.0001$ & 0.0086 & $<0.0001$ \\
\hline & Variance & $1.28 \times 10^{-3}$ & $4.16 \times 10^{-2}$ & $3.25 \times 10^{-2}$ & $1.46 \times 10^{-3}$ & $1.70 \times 10^{-2}$ \\
\hline \multirow{5}{*}{$\begin{array}{c}\text { ERBB2 } \\
\text { rs133724008 } \\
\text { g.873T }>C\end{array}$} & CC(119) & $10311 \pm 90.86^{\mathrm{a}}$ & $361.80 \pm 3.81^{\mathrm{a}}$ & $3.574 \pm 0.037$ & $324.73 \pm 2.78^{A}$ & $3.195 \pm 0.013^{\mathrm{A}}$ \\
\hline & СТ(412) & $10469 \pm 62.32^{\mathrm{ab}}$ & $365.97 \pm 2.60^{\mathrm{ab}}$ & $3.542 \pm 0.025$ & $327.50 \pm 1.89 \mathrm{~A}$ & $3.150 \pm 0.009^{\mathrm{B}}$ \\
\hline & ТT(471) & $10551 \pm 62.29^{b}$ & $370.49 \pm 2.60^{\mathrm{b}}$ & $3.595 \pm 0.025$ & $331.81 \pm 1.89^{\mathrm{B}}$ & $3.178 \pm 0.009^{\mathrm{A}}$ \\
\hline & $p$-value & 0.0178 & 0.0290 & 0.0666 & 0.0069 & $<0.0001$ \\
\hline & Variance & $1.49 \times 10^{-2}$ & $9.02 \times 10^{-3}$ & $9.40 \times 10^{-5}$ & $9.89 \times 10^{-3}$ & $1.78 \times 10^{-2}$ \\
\hline \multirow{5}{*}{$\begin{array}{c}E R B B 2 \\
\text { ss } 1996900615 \\
\text { g.20982del }\end{array}$} & DEL(372) & $10453 \pm 63.86$ & $366.24 \pm 2.66$ & $3.594 \pm 0.026$ & $327.94 \pm 1.94$ & $3.178 \pm 0.009^{a}$ \\
\hline & DEL.TC(471) & $10478 \pm 61.61$ & $367.51 \pm 2.57$ & $3.561 \pm 0.025$ & $328.49 \pm 1.87$ & $3.158 \pm 0.009^{b}$ \\
\hline & $\mathrm{TC}(162)$ & $10449 \pm 83.25$ & $366.96 \pm 3.49$ & $3.561 \pm 0.033$ & $328.21 \pm 2.54$ & $3.181 \pm 0.012^{\mathrm{a}}$ \\
\hline & $p$-value & 0.8756 & 0.8715 & 0.3157 & 0.9526 & 0.0140 \\
\hline & Variance & $5.87 \times 10^{-5}$ & $1.58 \times 10^{-5}$ & $9.68 \times 10^{-4}$ & $2.45 \times 10^{-6}$ & $1.60 \times 10^{-3}$ \\
\hline \multirow{5}{*}{$\begin{array}{c}\text { ERBB2 } \\
\text { rs110735562 } \\
\text { g.21561A>G }\end{array}$} & $\mathrm{AA}(65)$ & $10305 \pm 115.13^{\mathrm{A}}$ & $364.26 \pm 4.86^{\mathrm{AB}}$ & $3.592 \pm 0.046$ & $323.00 \pm 3.54^{\mathrm{A}}$ & $3.180 \pm 0.016$ \\
\hline & AG(362) & $10566 \pm 66.63^{B}$ & $372.41 \pm 2.78^{\mathrm{A}}$ & $3.577 \pm 0.027$ & $331.66 \pm 2.02^{B}$ & $3.171 \pm 0.009$ \\
\hline & GG(585) & $10410 \pm 58.40^{\mathrm{A}}$ & $363.35 \pm 2.43^{B}$ & $3.565 \pm 0.023$ & $325.84 \pm 1.77^{\mathrm{A}}$ & $3.165 \pm 0.008$ \\
\hline & $p$-value & 0.0060 & 0.0006 & 0.7432 & 0.0010 & 0.4977 \\
\hline & Variance & $1.79 \times 10^{-2}$ & $6.48 \times 10^{-3}$ & $9.48 \times 10^{-4}$ & $2.05 \times 10^{-2}$ & $2.37 \times 10^{-3}$ \\
\hline \multirow{5}{*}{$\begin{array}{c}\text { ERBB2 } \\
\text { rs109017161 } \\
\text { g.22268T>C }\end{array}$} & CC(164) & $10465 \pm 63.62$ & $367.53 \pm 2.66$ & $3.599 \pm 0.026$ & $328.83 \pm 1.94$ & $3.180 \pm 0.009^{\mathrm{A}}$ \\
\hline & CT(458) & $10465 \pm 61.90$ & $367.96 \pm 2.59$ & $3.569 \pm 0.025$ & $327.77 \pm 1.89$ & $3.157 \pm 0.009^{\mathrm{B}}$ \\
\hline & ТT(378) & $10422 \pm 83.31$ & $363.93 \pm 3.50$ & $3.549 \pm 0.034$ & $326.39 \pm 2.55$ & $3.178 \pm 0.012 \mathrm{AB}$ \\
\hline & $p$-value & 0.8382 & 0.4394 & 0.2187 & 0.5892 & 0.0062 \\
\hline & Variance & $6.05 \times 10^{-4}$ & $2.78 \times 10^{-3}$ & $3.24 \times 10^{-3}$ & $1.58 \times 10^{-3}$ & $5.71 \times 10^{-4}$ \\
\hline \multirow{5}{*}{$\begin{array}{c}E R B B 2 \\
\text { rs109122971 } \\
\text { g.23650T>C }\end{array}$} & CC(332) & $10457 \pm 66.19$ & $367.99 \pm 2.77$ & $3.608 \pm 0.027$ & $328.41 \pm 2.02$ & $3.179 \pm 0.009^{\mathrm{A}}$ \\
\hline & CT(473) & $10488 \pm 61.60$ & $368.74 \pm 2.57$ & $3.563 \pm 0.025$ & $328.80 \pm 1.87$ & $3.157 \pm 0.009^{\mathrm{B}}$ \\
\hline & ТT(196) & $10377 \pm 77.66$ & $363.34 \pm 3.26$ & $3.564 \pm 0.031$ & $325.82 \pm 2.37$ & $3.185 \pm 0.011^{\mathrm{A}}$ \\
\hline & $p$-value & 0.2843 & 0.1835 & 0.1368 & 0.3740 & 0.0038 \\
\hline & Variance & $2.32 \times 10^{-3}$ & $4.14 \times 10^{-3}$ & $2.09 \times 10^{-3}$ & $2.41 \times 10^{-3}$ & $1.86 \times 10^{-3}$ \\
\hline \multirow{5}{*}{$\begin{array}{c}E R B B 2 \\
\text { ss } 1996900614 \\
\text { g.19414A>G }\end{array}$} & $\mathrm{AA}(165)$ & $10381 \pm 82.85$ & $363.42 \pm 3.48$ & $3.565 \pm 0.033$ & $325.46 \pm 2.54$ & $3.181 \pm 0.012^{\mathrm{A}}$ \\
\hline & $\mathrm{AG}(470)$ & $10502 \pm 61.53$ & $368.89 \pm 2.57$ & $3.563 \pm 0.025$ & $329.13 \pm 1.87$ & $3.157 \pm 0.009^{B}$ \\
\hline & GG(381) & $10476 \pm 63.60$ & $368.21 \pm 2.66$ & $3.598 \pm 0.026$ & $329.29 \pm 1.94$ & $3.178 \pm 0.009^{\mathrm{A}}$ \\
\hline & $p$-value & 0.2745 & 0.2179 & 0.2796 & 0.2308 & 0.0078 \\
\hline & Variance & $3.64 \times 10^{-3}$ & $4.98 \times 10^{-3}$ & $8.93 \times 10^{-4}$ & $5.28 \times 10^{-3}$ & $1.75 \times 10^{-3}$ \\
\hline \multirow{5}{*}{$\begin{array}{c}\text { ERBB2 } \\
\text { rs110133654 } \\
\text { g.10727A>G }\end{array}$} & $\mathrm{AA}(372)$ & $10501 \pm 64.00$ & $369.19 \pm 2.68$ & $3.592 \pm 0.026$ & $330.40 \pm 1.95$ & $3.178 \pm 0.009 \mathrm{~A}$ \\
\hline & $\mathrm{AG}(470)$ & $10490 \pm 61.54$ & $366.60 \pm 2.56$ & $3.548 \pm 0.025$ & $328.63 \pm 1.87$ & $3.155 \pm 0.009^{B}$ \\
\hline & GG(163) & $10416 \pm 83.71$ & $364.56 \pm 3.52$ & $3.549 \pm 0.034$ & $327.67 \pm 2.56$ & $3.180 \pm 0.012^{\mathrm{A}}$ \\
\hline & $p$-value & 0.5360 & 0.3295 & 0.1291 & 0.4442 & 0.0049 \\
\hline & Variance & $2.20 \times 10^{-3}$ & $2.73 \times 10^{-3}$ & $1.57 \times 10^{-3}$ & $1.67 \times 10^{-3}$ & $1.62 \times 10^{-3}$ \\
\hline \multirow{5}{*}{$\begin{array}{c}E R B B 2 \\
\text { rs109941438 } \\
\text { g.11680C }>\mathrm{T}\end{array}$} & CC(375) & $10464 \pm 63.85$ & $367.66 \pm 2.67$ & $3.593 \pm 0.026$ & $329.19 \pm 1.94$ & $3.181 \pm 0.009 \mathrm{~A}$ \\
\hline & CT(452) & $10482 \pm 61.95$ & $367.23 \pm 2.58$ & $3.553 \pm 0.025$ & $328.40 \pm 1.88$ & $3.157 \pm 0.009^{\mathrm{B}}$ \\
\hline & ТT(166) & $10364 \pm 82.72$ & $360.55 \pm 3.47$ & $3.543 \pm 0.033$ & $324.45 \pm 2.53$ & $3.183 \pm 0.012^{\mathrm{A}}$ \\
\hline & $p$-value & 0.2884 & 0.0724 & 0.1341 & 0.1331 & 0.0034 \\
\hline & Variance & $3.78 \times 10^{-3}$ & $9.51 \times 10^{-3}$ & $2.76 \times 10^{-3}$ & $7.37 \times 10^{-3}$ & $1.57 \times 10^{-3}$ \\
\hline \multirow{5}{*}{$\begin{array}{c}\text { ERBB2 } \\
\text { rs110552983 } \\
\text { g.16431C>G }\end{array}$} & CC(164) & $10440 \pm 82.87$ & $366.09 \pm 3.48$ & $3.559 \pm 0.033$ & $327.50 \pm 2.53$ & $3.177 \pm 0.012 \mathrm{AB}$ \\
\hline & CG(472) & $10521 \pm 61.62$ & $368.52 \pm 2.58$ & $3.551 \pm 0.025$ & $330.20 \pm 1.88$ & $3.156 \pm 0.009^{\mathrm{A}}$ \\
\hline & GG(381) & $10511 \pm 63.46$ & $370.25 \pm 2.65$ & $3.598 \pm 0.026$ & $331.32 \pm 1.93$ & $3.179 \pm 0.009^{B}$ \\
\hline & $p$-value & 0.5463 & 0.4421 & 0.1045 & 0.2855 & 0.0080 \\
\hline & Variance & $1.90 \times 10^{-3}$ & $2.49 \times 10^{-3}$ & $1.07 \times 10^{-3}$ & $4.35 \times 10^{-3}$ & $5.06 \times 10^{-4}$ \\
\hline \multirow{5}{*}{$\begin{array}{c}\text { ERBB2 } \\
\text { rs133031530 } \\
\text { g.22346A }>\mathrm{T}\end{array}$} & AA(162) & $10420 \pm 84.37$ & $363.01 \pm 3.54$ & $3.539 \pm 0.034$ & $327.77 \pm 2.58$ & $3.182 \pm 0.012^{\mathrm{A}}$ \\
\hline & $\mathrm{AT}(445)$ & $10452 \pm 64.28$ & $364.58 \pm 2.68$ & $3.544 \pm 0.026$ & $327.28 \pm 1.95$ & $3.155 \pm 0.009^{B}$ \\
\hline & ТT(379) & $10457 \pm 65.19$ & $365.70 \pm 2.72$ & $3.587 \pm 0.026$ & $329.02 \pm 1.98$ & $3.181 \pm 0.009^{\mathrm{A}}$ \\
\hline & $p$-value & 0.8889 & 0.7097 & 0.1197 & 0.6089 & 0.0015 \\
\hline & Variance & $4.20 \times 10^{-4}$ & $1.04 \times 10^{-3}$ & $2.21 \times 10^{-3}$ & $1.45 \times 10^{-4}$ & $1.60 \times 10^{-3}$ \\
\hline \multirow{5}{*}{$\begin{array}{c}E R B B 2 \\
\text { rs109763505 } \\
\text { g.22400A>G }\end{array}$} & AA(195) & $10476 \pm 77.76$ & $368.62 \pm 3.26$ & $3.567 \pm 0.031$ & $330.12 \pm 2.38$ & $3.182 \pm 0.011^{\mathrm{a}}$ \\
\hline & AG(483) & $10515 \pm 61.66$ & $369.25 \pm 2.57$ & $3.563 \pm 0.025$ & $330.07 \pm 1.87$ & $3.160 \pm 0.009^{b}$ \\
\hline & GG(332) & $10495 \pm 66.45$ & $369.09 \pm 2.78$ & $3.606 \pm 0.027$ & $329.97 \pm 2.03$ & $3.180 \pm 0.009^{a}$ \\
\hline & $p$-value & 0.8460 & 0.9776 & 0.1747 & 0.9973 & 0.0177 \\
\hline & Variance & $1.65 \times 10^{-4}$ & $4.54 \times 10^{-5}$ & $1.50 \times 10^{-3}$ & $5.58 \times 10^{-6}$ & $6.97 \times 10^{-4}$ \\
\hline
\end{tabular}

Note: $p$-value refers to the results of association analysis between each SNP and milk production traits. Different letter (small letters: $p<0.05$; capital letters: $p<0.01$ ) superscripts (adjusted value after correction for multiple testing) indicate significant differences among the genotypes. 


\subsection{LD and Haplotypes Analyses}

One SNP (ss1996900615) identified for ERBB2 gene was insertion/deletion (InDel), whereas the remaining 10 SNPs were used to perform LD analysis. Pair-wise $\mathrm{D}^{\prime}$ measures showed that nine SNPs in ERBB2 were highly linked $\left(\mathrm{D}^{\prime}=0.99 \sim 1.00\right)$ and one $12 \mathrm{~Kb}$ haplotype block comprising these nine SNPs were inferred (Figure 1), in which three main haplotypes were formed. The frequencies of the haplotypes ACGGGCTGC, GTCAATAAT, and GTCAGTAAT were 56.57\%, 24.15\%, and 15.04\%, respectively (Table 4). Subsequently, haplotype-based analysis showed significant association of the haplotypes with all four milk production traits, except the milk yield ( $p=0.0130$ to $p<0.0001$, Table 5 ). No LD was observed between the two identified SNPs for HSPA8 gene.

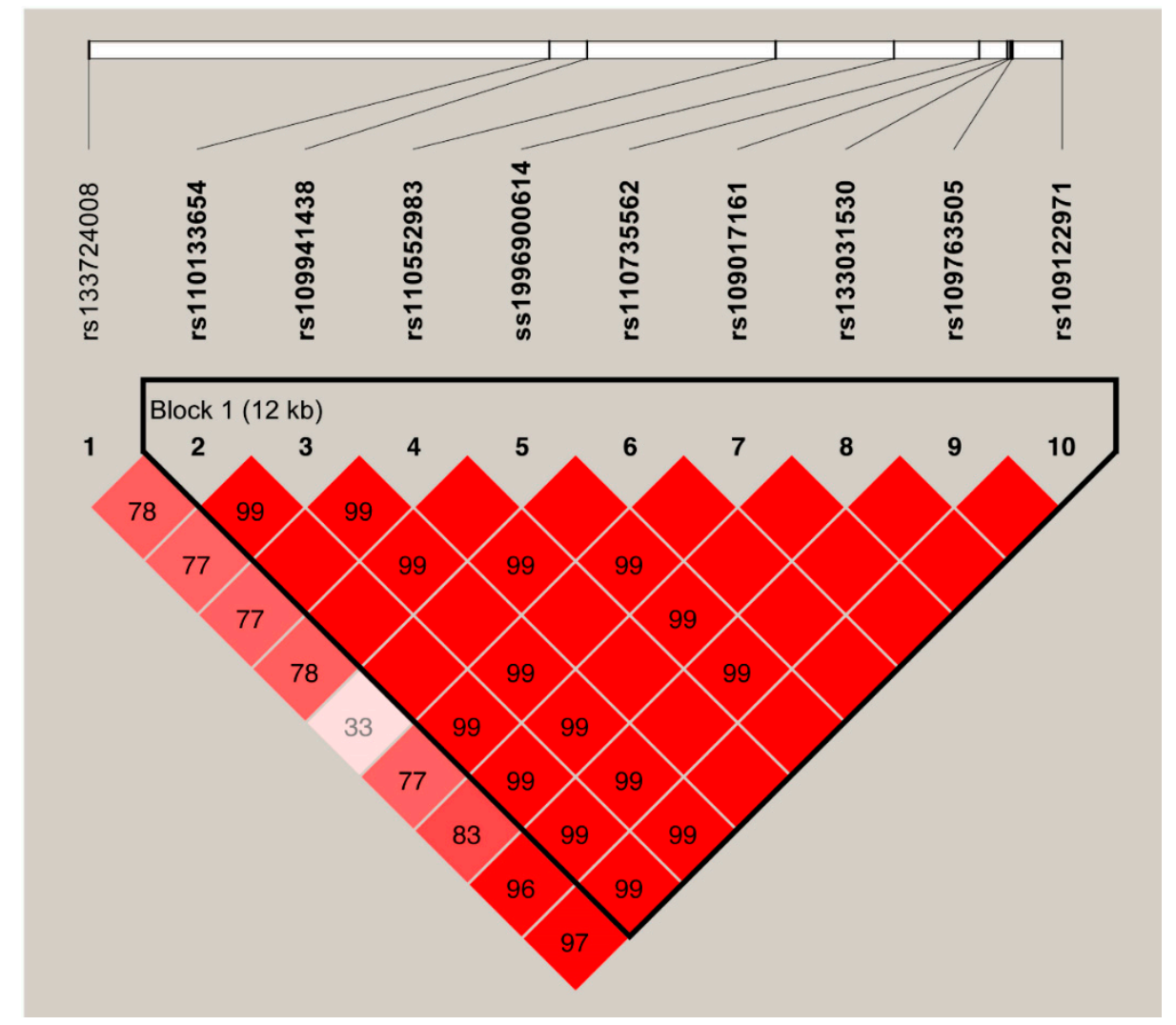

Figure 1. The haplotype blocks and pairwise linkage disequilibrium (LD) values $\left(\mathrm{D}^{\prime}\right)$ for the ten SNPs in ERBB2. Legends: The values within boxes are pair wise SNP correlation $\left(\mathrm{D}^{\prime}\right)$, bright red boxes without numbers indicate complete $\mathrm{LD}\left(\mathrm{D}^{\prime}=1\right)$. The brighter shade of red indicates higher LD.

Table 4. Main haplotypes and their frequencies observed in ERBB2 gene.

\begin{tabular}{ccccccccccc}
\hline $\begin{array}{c}\text { ERBB2 } \\
\text { Haplotypes }\end{array}$ & $\begin{array}{c}\text { SNP1 } \\
\text { G > A }\end{array}$ & $\begin{array}{c}\text { SNP2 } \\
\text { T > C }\end{array}$ & $\begin{array}{c}\text { SNP3 } \\
\text { C }>\text { G }\end{array}$ & $\begin{array}{c}\text { SNP4 } \\
\text { A }>\text { G }\end{array}$ & $\begin{array}{c}\text { SNP5 } \\
\text { A }>\text { G }\end{array}$ & $\begin{array}{c}\text { SNP6 } \\
\text { T }>\text { C }\end{array}$ & $\begin{array}{c}\text { SNP7 } \\
\text { A }>\text { T }\end{array}$ & $\begin{array}{c}\text { SNP8 } \\
\text { A }>\text { G }\end{array}$ & $\begin{array}{c}\text { SNP9 } \\
\text { T }>\text { C }\end{array}$ & $\begin{array}{c}\text { Frequency } \\
\text { (\%) }\end{array}$ \\
\hline ACGGGCTGC & A & C & G & G & G & C & T & G & C & 56.57 \\
GTCAATAAT & G & T & C & A & A & T & A & A & T & 24.15 \\
GTCAGTAAT & G & T & C & A & G & T & A & A & T & 15.04 \\
\hline
\end{tabular}

Note: The Ref number of each SNP can be found in the haplotype Figure 1. SNP1 = rs110133654, SNP2 = rs109941438, SNP3 = rs110552983, SNP4 = ss1996900614, SNP5 = rs110735562, SNP6 = rs109017161, SNP7 = rs133031530, SNP8 = rs109763505, SNP9 $=$ rs109122971. 
Table 5. Haplotype associations of the nine SNPs in ERBB2 with milk production traits in Chinese Holstein cattle $(\mathrm{LSM} \pm \mathrm{SE})$.

\begin{tabular}{cccccc}
\hline $\begin{array}{c}\text { ERBB2 } \\
\text { Haplotypes }\end{array}$ & Milk Yield & Fat Yield & Fat Percentage & Protein Yield & $\begin{array}{c}\text { Protein } \\
\text { Percentage }\end{array}$ \\
\hline $\mathrm{H} 1 \mathrm{H} 1(337)$ & $10464 \pm 70.84$ & $366.88 \pm 2.97^{\mathrm{AC}}$ & $3.605 \pm 0.029^{\mathrm{a}}$ & $327.87 \pm 2.16^{\mathrm{A}}$ & $3.178 \pm 0.010^{\mathrm{A}}$ \\
$\mathrm{H} 1 \mathrm{H} 2(272)$ & $10536 \pm 76.34$ & $371.71 \pm 3.19^{\mathrm{A}}$ & $3.603 \pm 0.031^{\mathrm{a}}$ & $330.23 \pm 2.33^{\mathrm{A}}$ & $3.174 \pm 0.011^{\mathrm{A}}$ \\
$\mathrm{H} 1 \mathrm{H} 3(169)$ & $10348 \pm 82.03$ & $355.82 \pm 3.45^{\mathrm{B}}$ & $3.507 \pm 0.033^{\mathrm{b}}$ & $320.29 \pm 2.51^{\mathrm{B}}$ & $3.130 \pm 0.011^{\mathrm{B}}$ \\
$\mathrm{H} 2 \mathrm{H} 2(65)$ & $10296 \pm 117.42$ & $362.97 \pm 4.96^{\mathrm{AB}}$ & $3.593 \pm 0.047^{\mathrm{ab}}$ & $322.42 \pm 3.61^{\mathrm{AB}}$ & $3.183 \pm 0.016^{\mathrm{A}}$ \\
$\mathrm{H} 2 \mathrm{H} 3(82)$ & $10416 \pm 108.62$ & $357.93 \pm 4.57^{\mathrm{BC}}$ & $3.521 \pm 0.044^{\mathrm{ab}}$ & $323.81 \pm 3.33^{\mathrm{AB}}$ & $3.183 \pm 0.015^{\mathrm{A}}$ \\
$\mathrm{H} 3 \mathrm{H} 3(19)$ & $10122 \pm 203.08$ & $360.35 \pm 8.60^{\mathrm{AB}}$ & $3.612 \pm 0.082^{\mathrm{ab}}$ & $319.76 \pm 6.27^{\mathrm{AB}}$ & $3.180 \pm 0.028^{\mathrm{AB}}$ \\
$p$-value & 0.0538 & $<0.0001$ & 0.0130 & $0.0009^{2}$ & 0.0001 \\
\hline
\end{tabular}

Note: $p$-value refers to the results of association analysis between each haplotype and milk production traits. Different letter (small letters: $p<0.05$; capital letters: $p<0.01$ ) superscripts (adjusted value after correction for multiple testing) indicate significant differences among the haplotypes. H1 = ACGGGCTGC, H2 = GTCAATAAT, H3 = GTCAGTAAT.

\section{Discussion}

The results of the present study confirmed the significant genetic effects of HSPA8 and ERBB2 genes on milk protein traits in a large population of dairy cattle. The two candidate genes also had remarkable impacts on other milk production traits, which may provide new insights into the enhancement of milk profiles via selection strategies.

Two SNPs, rs110735562 and rs109017161 in exonic region of ERBB2 showed significant associations with milk protein traits are synonymous variations, which could modify mRNA stability and further affect protein expression [24,25]. The SNPs have affect the promoter activity and gene expression [26], and a previous study reported that two SNPs (rs209535817 and rs210440016) in the $5^{\prime}$-UTR region of bovine $S A A 2$ (serum amyloid A2) gene impact the phenotype through altering the promoter activity [27]. Hence, it suggested that the genetic effect of SNP rs133724008 identified in the 5'-UTR of the $E R B B 2$ gene on milk production traits was likely due to the impacts on its transcription. Generally, mature miRNAs are bound to mRNA ribosomal complex and regulate the expression of target genes by complementary recognition of the $3^{\prime}$-UTR of the mRNA $[28,29]$. Thus, whether there are relative miRNAs binding to the $3^{\prime}$-UTR (Position: Chr15, 34216278-34216505) of HSPA8 including identified SNPs were predicted by RNAhybrid software [30]. The results showed that miR-301a was targeted to the 3'-UTR including SNP rs136632043 (Position: Chr15, 34216487, Table S4), which indicated the significant associations of SNP rs136632043 in $3^{\prime}$-UTR of HSPA8 with milk production traits probably resulted from miR-301a or unknown potential regulatory mechanism. Although an intron does not hold a sequence for coding protein, an important function of SNPs in introns in altering gene transcriptional level has been elucidated [31,32]. Additionally, the significant associations between SNPs in introns with milk protein traits are also likely due to their LD with true causative variation.

As a group of highly conserved and widely expressed proteins, heat-shock proteins (HSPs) play important physiological functions [33]. For example, HSPA8 has been validated as an evolutionarily conserved protein in swine and bovine [34]. HSPA8 is highly involved in many biological processes, including proteasomal degradation [35], catalyzing protein folding and clathrin uncoating [36], and other protein networks involved in protein catabolism, protein homeostasis, ubiquitination, carbohydrate metabolism and cell cycle control [37]. ERBB2 is a member of a family of transmembrane receptor tyrosine kinases involved in the regulation of cellular processes by modulation of several pathways, such as mTOR, MAPK, and PI3K/AKT pathways [38-40]. In consistent with the present study, the functional role of ERBB2 on milk production traits has been also identified as positional candidate gene for lactation persistency in Canadian Holstein cattle [41]. Therefore, the results of the current study and previously published research indicate that HSPA 8 and ERBB2 are promising candidate genes that have strong genetic effects on milk production traits. In addition, both single SNP-based and haplotype-based association analyses also suggest that the novel SNPs in these two genes may be used as potential genetic markers for genetic improvement in dairy breeding schemes. 
Generally, a small proportion with less than $1 \%$ of the phenotypic variance was explained by polymorphisms underlying complex traits in livestock animals [42]. In the present study, six and four significant pairs of SNP-trait explaining phenotypic variations with greater than $1 \%$ were found in HSPA 8 and ERBB2, respectively. These results suggest that the subset of these large-effect SNPs (rs132976221, rs136632043, rs133724008, and rs110735562) could be used as potential genetic markers for further marker-assisted selection (MAS) in milk production traits, especially for milk protein traits. All identified SNPs in HSPA8 and ERBB2 genes could be incorporated into the SNP panels for genomic selection of dairy cattle breeding schemes and could be used to improve frequencies of the genetic markers that are positively related to milk production traits of interest.

Supplementary Materials: The following are available online at http:/ /www.mdpi.com/2073-4425/10/2/104/s1. Table S1: The distribution of daughters from 17 herds in 17 farms. Table S2: PCR primers information of HSPA8 and ERBB2 genes. Table S3: Additive, dominant and allele substitution effects of the SNPs associated with milk production traits of HSPA8 and ERBB2 in Chinese Holstein cattle. Table S4: The predicted miRNAs targeted to the 3'-UTR of HSPA8 gene.

Author Contributions: C.L. and M.W. conducted association analysis and wrote the manuscript. W.C. and S.L. took part in SNP identification and genotyping. C.Z. and H.Y. collected phenotypes and pedigree data. D.S. participated in the data filtering and provided suggestions for the manuscript. S.Z. designed the study and revised the manuscript. All authors read and approved the final manuscript.

Funding: This work was supported by the Young Talent Fund of University Association for Science and Technology in Shaanxi, China (20170202), Postdoctoral Science Foundation of Shaanxi (2017BSHEDZZ107), and Ph.D research start-up foundation of Northwest A\&F University (2452016203).

Acknowledgments: We appreciate the Dairy Data Center of China and Beijing Dairy Cattle Center for providing pedigree and DHI data for the Chinese Holstein cows. The authors are grateful to Juan J. Loor from University of Illinois for proofreading the manuscript.

Conflicts of Interest: The authors declare no conflict of interest, financial or otherwise.

\section{References}

1. Chen, H.Y.; Zhang, Q.; Yin, C.C.; Wang, C.K.; Gong, W.J.; Mei, G. Detection of quantitative trait loci affecting milk production traits on bovine chromosome 6 in a Chinese Holstein population by the daughter design. J. Dairy Sci. 2006, 89, 782-790. [CrossRef]

2. Kolbehdari, D.; Wang, Z.; Grant, J.R.; Murdoch, B.; Prasad, A.; Xiu, Z.; Marques, E.; Stothard, P.; Moore, S.S. A whole genome scan to map QTL for milk production traits and somatic cell score in Canadian Holstein bulls. J. Anim. Breed. Genet. 2009, 126, 216-227. [CrossRef]

3. Fontanesi, L.; Calo, D.G.; Galimberti, G.; Negrini, R.; Marino, R.; Nardone, A.; Ajmone-Marsan, P.; Russo, V. A candidate gene association study for nine economically important traits in Italian Holstein cattle. Anim. Genet. 2014, 45, 576-580. [CrossRef] [PubMed]

4. Huang, W.; Penagaricano, F.; Ahmad, K.R.; Lucey, J.A.; Weigel, K.A.; Khatib, H. Association between milk protein gene variants and protein composition traits in dairy cattle. J. Dairy Sci. 2012, 95, 440-449. [CrossRef] [PubMed]

5. Molee, A.; Poompramun, C.; Mernkrathoke, P. Effect of casein genes - beta-LGB, DGAT1, GH, and LHR - on milk production and milk composition traits in crossbred Holsteins. Genet. Mol. Res. 2015, 14, 2561-2571. [CrossRef] [PubMed]

6. Raven, L.A.; Cocks, B.G.; Kemper, K.E.; Chamberlain, A.J.; Vander Jagt, C.J.; Goddard, M.E.; Hayes, B.J. Targeted imputation of sequence variants and gene expression profiling identifies twelve candidate genes associated with lactation volume, composition and calving interval in dairy cattle. Mamm. Genome Off. J. Int. Mamm. Genome Soc. 2016, 27, 81-97. [CrossRef] [PubMed]

7. Sanchez, M.P.; Wolf, V.; El Jabri, M.; Beuvier, E.; Rolet-Repecaud, O.; Gauzere, Y.; Minery, S.; Brochard, M.; Michenet, A.; Taussat, S.; et al. Short communication: Confirmation of candidate causative variants on milk composition and cheesemaking properties in Montbeliarde cows. J. Dairy Sci. 2018, 101, 10076-10081. [CrossRef] [PubMed] 
8. Schopen, G.C.; Visker, M.H.; Koks, P.D.; Mullaart, E.; van Arendonk, J.A.; Bovenhuis, H. Whole-genome association study for milk protein composition in dairy cattle. J. Dairy Sci. 2011, 94, 3148-3158. [CrossRef] [PubMed]

9. Zhou, H.; Cheng, L.; Azimu, W.; Hodge, S.; Edwards, G.R.; Hickford, J.G. Variation in the bovine FABP4 gene affects milk yield and milk protein content in dairy cows. Sci. Rep. 2015, 5, 10023. [CrossRef]

10. Li, C.; Cai, W.; Zhou, C.; Yin, H.; Zhang, Z.; Loor, J.J.; Sun, D.; Zhang, Q.; Liu, J.; Zhang, S. RNA-Seq reveals 10 novel promising candidate genes affecting milk protein concentration in the Chinese Holstein population. Sci. Rep. 2016, 6, 26813. [CrossRef]

11. Bionaz, M.; Loor, J.J. Gene networks driving bovine mammary protein synthesis during the lactation cycle. Bioinform. Biol. Insights 2011, 5, 83-98. [CrossRef] [PubMed]

12. Proud, C.G. Signalling to translation: How signal transduction pathways control the protein synthetic machinery. Biochem. J. 2007, 403, 217-234. [CrossRef] [PubMed]

13. Paten, A.M.; Duncan, E.J.; Pain, S.J.; Peterson, S.W.; Kenyon, P.R.; Blair, H.T.; Dearden, P.K. Functional development of the adult ovine mammary gland-Insights from gene expression profiling. BMC Genom. 2015, 16, 748. [CrossRef] [PubMed]

14. Lee, A.S. Glucose-regulated proteins in cancer: Molecular mechanisms and therapeutic potential. Nat. Rev. Cancer 2014, 14, 263-276. [CrossRef] [PubMed]

15. Coussens, L.; Yang-Feng, T.L.; Liao, Y.C.; Chen, E.; Gray, A.; McGrath, J.; Seeburg, P.H.; Libermann, T.A.; Schlessinger, J.; Francke, U.; et al. Tyrosine kinase receptor with extensive homology to EGF receptor shares chromosomal location with neu oncogene. Science 1985, 230, 1132-1139. [CrossRef] [PubMed]

16. Anderson, S.M.; Rudolph, M.C.; McManaman, J.L.; Neville, M.C. Key stages in mammary gland development. Secretory activation in the mammary gland: it's not just about milk protein synthesis! Breast Cancer Res. 2007, 9, 204. [CrossRef] [PubMed]

17. Ciocca, D.R.; Cuello-Carrion, F.D.; Natoli, A.L.; Restall, C.; Anderson, R.L. Absence of caveolin-1 alters heat shock protein expression in spontaneous mammary tumors driven by Her-2/neu expression. Histochem. Cell Biol. 2012, 137, 187-194. [CrossRef]

18. Yue, L.U.; Xiang, J.Y.; Sun, P.; Yao, Y.S.; Sun, Z.N.; Liu, X.P.; Wang, H.B.; Shen, Z.; Yao, R.Y. Relationship between HSP70 and ERBB2 expression in breast cancer cell lines regarding drug resistance. Anticancer Res. 2016, 36, 1243-1249.

19. Li, C.; Sun, D.; Zhang, S.; Yang, S.; Alim, M.A.; Zhang, Q.; Li, Y.; Liu, L. Genetic effects of FASN, PPARGC1A, ABCG2 and IGF1 revealing the association with milk fatty acids in a Chinese Holstein cattle population based on a post genome-wide association study. BMC Genet. 2016, 17, 110. [CrossRef]

20. Barrett, J.C.; Fry, B.; Maller, J.; Daly, M.J. Haploview: Analysis and visualization of LD and haplotype maps. Bioinformatics 2005, 21, 263-265. [CrossRef]

21. Browning, S.R.; Browning, B.L. Rapid and accurate haplotype phasing and missing-data inference for whole-genome association studies by use of localized haplotype clustering. Am. J. Hum. Genet. 2007, 81, 1084-1097. [CrossRef] [PubMed]

22. Gabriel, S.B.; Schaffner, S.F.; Nguyen, H.; Moore, J.M.; Roy, J.; Blumenstiel, B.; Higgins, J.; DeFelice, M.; Lochner, A.; Faggart, M.; et al. The structure of haplotype blocks in the human genome. Science 2002, 296, 2225-2229. [CrossRef] [PubMed]

23. Falconer, D.S.; Mackay, T.F.C. Introduction to Quantitative Genetics, 4th ed.; Longman Scientific and Technical: New York, NY, USA, 1996.

24. Capon, F.; Allen, M.H.; Ameen, M.; Burden, A.D.; Tillman, D.; Barker, J.N.; Trembath, R.C. A synonymous SNP of the corneodesmosin gene leads to increased mRNA stability and demonstrates association with psoriasis across diverse ethnic groups. Hum. Mol. Genet. 2004, 13, 2361-2368. [CrossRef]

25. Nackley, A.G.; Shabalina, S.A.; Tchivileva, I.E.; Satterfield, K.; Korchynskyi, O.; Makarov, S.S.; Maixner, W.; Diatchenko, L. Human catechol-O-methyltransferase haplotypes modulate protein expression by altering mRNA secondary structure. Science 2006, 314, 1930-1933. [CrossRef] [PubMed]

26. Huang, Y.Z.; Li, M.X.; Wang, J.; Zhan, Z.Y.; Sun, Y.J.; Sun, J.J.; Li, C.J.; Lan, X.Y.; Lei, C.Z.; Zhang, C.L.; et al. A 5 '-regulatory region and two coding region polymorphisms modulate promoter activity and gene expression of the growth suppressor gene ZBED6 in cattle. PLOS ONE 2013, 8. [CrossRef] [PubMed] 
27. Yang, S.; Li, C.; Xie, Y.; Cui, X.; Li, X.; Wei, J.; Zhang, Y.; Yu, Y.; Wang, Y.; Zhang, S.; et al. Detection of functional polymorphisms influencing the promoter activity of the $S A A 2$ gene and their association with milk production traits in Chinese Holstein cows. Anim. Genet. 2015, 46, 591-598. [CrossRef] [PubMed]

28. He, J.P.; Feng, X.; Hua, J.R.; Wei, L.; Lu, Z.W.; Wei, W.J.; Cai, H.; Wang, B.; Shi, W.G.; Ding, N.; et al. miR-300 regulates cellular radiosensitivity through targeting p53 and apaf1 in human lung cancer cells. Cell Cycle 2017, 16, 1943-1953. [CrossRef] [PubMed]

29. Wu, C.R.; Liu, Z.; Ma, L.; Pei, C.; Qin, L.; Gao, N.; Li, J.; Yin, Y. MiRNAs regulate oxidative stress related genes via binding to the $3^{\prime}$ UTR and TATA-box regions: A new hypothesis for cataract pathogenesis. BMC Ophthalmol. 2017, 17. [CrossRef] [PubMed]

30. Kruger, J.; Rehmsmeier, M. RNAhybrid: microRNA target prediction easy, fast and flexible. Nucleic Acids Res. 2006, 34, W451-W454. [CrossRef] [PubMed]

31. Ozaki, K.; Ohnishi, Y.; Iida, A.; Sekine, A.; Yamada, R.; Tsunoda, T.; Sato, H.; Sato, H.; Hori, M.; Nakamura, Y.; et al. Functional SNPs in the lymphotoxin-alpha gene that are associated with susceptibility to myocardial infarction. Nat. Genet. 2002, 32, 650-654. [CrossRef]

32. Tokuhiro, S.; Yamada, R.; Chang, X.T.; Suzuki, A.; Kochi, Y.; Sawada, T.; Suzuki, M.; Nagasaki, M.; Ohtsuki, M.; Ono, M.; et al. An intronic SNP in a RUNX1 binding site of SLC22A4, encoding an organic cation transporter, is associated with rheumatoid arthritis. Nat. Genet. 2003, 35, 341-348. [CrossRef] [PubMed]

33. Sun, H.; Cai, X.Y.; Zhou, H.F.; Li, X.Q.; Du, Z.P.; Zou, H.Y.; Wu, J.Y.; Xie, L.; Cheng, Y.W.; Xie, W.M.; et al. The protein-protein interaction network and clinical significance of heat-shock proteins in esophageal squamous cell carcinoma. Amino Acids 2018, 50, 685-697. [CrossRef] [PubMed]

34. Elliott, R.M.A.; Lloyd, R.E.; Fazeli, A.; Sostaric, E.; Georgiou, A.S.; Satake, N.; Watson, P.F.; Holt, W.V. Effects of HSPA8, an evolutionarily conserved oviductal protein, on boar and bull spermatozoa. Reproduction 2009, 137, 191-203. [CrossRef] [PubMed]

35. Bercovich, B.; Stancovski, I.; Mayer, A.; Blumenfeld, N.; Laszlo, A.; Schwartz, A.L.; Ciechanover, A. Ubiquitin-dependent degradation of certain protein substrates in vitro requires the molecular chaperone Hsc70. J. Biol. Chem. 1997, 272, 9002-9010. [CrossRef] [PubMed]

36. Newmyer, S.L.; Schmid, S.L. Dominant-interfering hsc70 mutants disrupt multiple stages of the clathrin-coated vesicle cycle in vivo. J. Cell Biol. 2001, 152, 607-620. [CrossRef] [PubMed]

37. Valek, L.; Heidler, J.; Scheving, R.; Wittig, I.; Tegeder, I. Nitric oxide contributes to protein homeostasis by S-nitrosylations of the chaperone HSPA8 and the ubiquitin ligase UBE2D. Redox Biol. 2018, 20, 217-235. [CrossRef] [PubMed]

38. Hynes, N.E.; Lane, H.A. ERBB receptors and cancer: The complexity of targeted inhibitors. Nat. Rev. Cancer 2005, 5, 341-354. [CrossRef] [PubMed]

39. Okines, A.; Cunningham, D.; Chau, I. Targeting the human EGFR family in esophagogastric cancer. Nat. Rev. Clin. Oncol. 2011, 8, 492-503. [CrossRef]

40. Li, N.; Han, M.; Zhou, N.; Tang, Y.; Tang, X.S. MicroRNA-495 confers increased sensitivity to chemotherapeutic agents in gastric cancer via the mammalian target of rapamycin (mTOR) signaling pathway by interacting with human epidermal growth factor receptor 2 (ERBB2). Med. Sci. Monit. Int. Med. J. Exp. Clin. Res. 2018, 24, 5960-5972. [CrossRef]

41. Do, D.N.; Bissonnette, N.; Lacasse, P.; Miglior, F.; Sargolzaei, M.; Zhao, X.; Ibeagha-Awemu, E.M. Genome-wide association analysis and pathways enrichment for lactation persistency in Canadian Holstein cattle. J. Dairy Sci. 2017, 100, 1955-1970. [CrossRef]

42. Wang, H.F.; Jiang, L.; Liu, X.; Yang, J.; Wei, J.L.; Xu, J.G.; Zhang, Q.; Liu, J.F. A post-GWAS replication study confirming the PTK2 gene associated with milk production traits in Chinese Holstein. PLoS ONE 2013, 8. [CrossRef] [PubMed]

(C) 2019 by the authors. Licensee MDPI, Basel, Switzerland. This article is an open access article distributed under the terms and conditions of the Creative Commons Attribution (CC BY) license (http:/ / creativecommons.org/licenses/by/4.0/). 